\title{
Nidotópica y parámetros reproductivos en el pico dorsiblanco Dendrocopos leucotos lilfordi Sharpe \& Dresser, 1871 en Navarra.
}

\author{
Nest site selection and reproduction parameters \\ in the White-backed Woodpecker Dendrocopos \\ leucotos lilfordi Sharpe \& Dresser, 1871 in Navarre.
}

Susana Cárcamo ${ }^{1}$, Miguel M. Elosegi ${ }^{2}$, Alfonso Senosiain ${ }^{3}$, Juan Arizaga ${ }^{4}$

(*)

\section{Resumen}

La población de pico dorsiblanco Dendrocopos leucotos lilfordi Sharpe \& Dresser, 1871 en España se estima en torno a las 100 parejas, el 98\% de la cual se localiza en Navarra. Gracias al seguimiento de la nidificación de la especie en Navarra durante los últimos años, se han podido recolectar gran cantidad de datos sobre el sustrato de nidificación y parámetros reproductivos. El objetivo de este artículo es (1) actualizar y contribuir al conocimiento de la nidotópica de la especie en Navarra, (2) describir su reproducción (tamaño de puesta y razón de sexos de la nidada) y (3) proponer, en relación a los resultados que se exponen, medidas de gestión útiles para su conservación. En cuanto al sustrato de nidificación ( $n=56$ pies), todos los nidos se localizaron en haya, la mayoría de ellos en pies puntisecos o totalmente verdes (46\%), seguido de árboles secos (40\%) y, finalmente y más rara vez, hayas mitad secas-mitad sanas (14\%). En promedio, la altura de los pies con nido fue $19,1 \pm 8,1 \mathrm{~m}$ y la altura de los nidos fue de 11,1 $\pm 4,2 \mathrm{~m}$. En promedio, la altitud de los nidos se situó a 943,8 \pm 99,0 m. s. n. m. y la orientación de los nidos fue heterogénea, siendo en este caso el promedio de $180 \pm 92^{\circ}$. La productividad se estimó en 1,4 $\pm 0,7$ pollos/nido (rango $=0-3 ; n=40$ ). La productividad no varió entre las dos zonas (Belate y

\footnotetext{
1 Bioma Forestal,

Polígono Parque Empresarial La Muga, 9, oficina 5, 31160 Orcoyen, Navarra.

2 Departamento de Medio Ambiente, Gobierno de Navarra. Calle González Tablas, 9, 31003 Pamplona, Navarra

3 alfonsocsenosiain@gmail.com

4 Departamento de Ornitología, Sociedad de Ciencias Aranzadi Zorroagagaina 11, 20014 Donostia.
}

*Correspondencia: susana@biomaforestal.es 
Quinto Real) en las que se obtuvo un tamaño muestral suficiente. La fecha media de salto de los pollos del nido fue el 28.05 ( \pm 8 días). La razón de sexos en pollos no varió significativamente de un ratio 1:1. Se recomienda minimizar las molestias entre los meses de marzo y junio, mantener en pie los árboles con nidos viejos, no realizar cortas en torno al nido, mantener la madera muerta sin eliminar los pies muertos o dañados, tanto si están en posición vertical como caídos y con independencia del diámetro.

Palabras clave: Picidae, madera muerta, gestión del hábitat, gestión forestal, especies amenazadas.

\section{Abstract}

The population of the White-backed Woodpecker Dendrocopos leucotos liffordi Sharpe \& Dresser, 1871 in Spain is estimated at around 100 pairs, 98\% of which are located in Navarre. By monitoring the breeding pairs of the species in Navarra over the last few years, it has been possible to collect a large amount of data on the nesting substrate and reproductive parameters. The objective of this article is (1) to update and contribute to the knowledge of the nidotopic (the type and location of the nesting tree) of the species in Navarra, (2) to describe its reproduction (laying size and sex ratio of the clutch) and (3) to propose, in relation to the results set out in this paper, a number of specific management measures that will be useful for its conservation. Regarding the nesting substrate $(n=56$ trees), all the nests were located in beech, most of them in trees with dry branches, or totally green trees (46\%), followed by dry trees (40\%) and, finally and more unusually, half dried-half healthy (14\%). On average, the mean height of the nesting tree was 19.1 $\pm 8.1 \mathrm{~m}$ and the height of the nests was $11.1 \pm 4.2 \mathrm{~m}$. On average, the altitude of the nests was located at $943.8 \pm 99.0$ meters above sea level, and the orientation of the nests was heterogeneous, in this case the average was between $180 \pm 92^{\circ}$. Productivity was estimated at $1.4 \pm 0.7$ chicks / nest (range $=0-3 ; n=40$ ). The productivity did not change between the two zones (Belate and Quinto Real) in which a sufficient sample size was obtained. The average fledgling date of the chicks was 28.05 ( \pm 8 days). The sex ratio in chickens did not vary significantly from a 1:1 ratio. It is recommended to minimize disturbance between March and June, keep trees with old nests standing, not to cut around the nest, keep dead wood without removing dead or damaged trees, both when these are standing and on the ground and regardless of the diameter.

Key words: Woodpeckers, dead wood, habitat management, forest management, endangered species.

\section{Laburpena}

Okil gibelnabarraren Dendrocopos leucotos lilfordi Sharpe \& Dresser, 1871 populazioa Espainian 100 bikote ingurukoa dela uste da, eta horietatik \%98 Nafarroan daude. Azken urteotan Nafarroan egin den habien jarraipenari esker datu ugari bildu ahal izan dira habiaegite substratuaz eta ugalketa parametroez. 
Artikulu honen helburuak dira (1) espezieak Nafarroan nolako habia-egite substratua duen hobeki ezagutzea eta eguneratzea, (2) beraren ugalketa deskribatzea (zenbateko errutea eta habiako sexu banaketa) eta (3), adierazitako datuei loturik, kontserbaziorako neurri eraginkorrak proposatzea.

Habia egiteko substratuari dagokionez ( $n=56$ oin), habia guztiak pagoetan aurkitu ziren, gehienak adar-mutur lehorrak edo guztiz berdeak (\%46), ondoren zuhaitz lehorrak (\%40) eta, azkenik, baten batzuk erdi lehor-erdi berde (\%14). Batezbeste, habia egiteko oinek

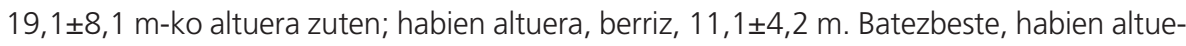
ra 943,8 eta 99,0 m-koa, eta habien orientazioa heterogeneoa, kasu honetan batezbestekoa $180 \pm 92^{\circ}$. Habietako produktibitatea 1,4 40,7 txita/habiako (rangoa $=0-3 ; n=40$ ). Produktibitatea ez zen aldatu lagin kopuru nahikoa lortutako bi eremuen artean (Belate eta Quinto Real). Txitek habiatik irteteko epea, 28.05 ( \pm 8 egun). Txitetan sexu banaketa ez zen askorik aldendua 1:1 ratiotik. Gomendioen artean, martxoa-ekaina bitartean ahalik eta gutxien molestatzea, habia zaharrak dituzten zuhaitzak ez botatzea, habia inguruetan mozketarik ez egitea, egur hila uztea oin hilak edo hondatuak kendu gabe, berdin zutik edo erorita egon eta diametroa ere kontuan hartu gabe.

Gako hitzak: Picidae, egur hila, habitataren gestioa, baso gestioa, espezie mehatxatuak.

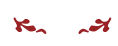

\section{Introducción}

Los pícidos constituyen un grupo de aves forestales de gran interés desde un punto de vista del ecosistema, ya que se consideran "arquitectos del paisaje" al contribuir a la creación de recursos que posteriormente son utilizados por un buen número de especies (Sekercioglu, 2006). Además, algunos pícidos se encuentran amenazados, por lo que tienen también interés para la conservación y gestión (Garmendia et al., 2006, Roberge et al., 2008, Cockle et al., 2011).

El pico dorsiblanco Dendrocopos leucotos lilfordi Sharpe \& Dresser, 1871 es un pícido eminentemente forestal asociado a bosques de caducifolios (principalmente hayedos) en los que se dan procesos de decadencia y abunda la madera muerta (Cramp, 1985). Su distribución global abarca casi todo el Paleártico templado y boreal, desde Europa hasta Asia oriental (Ilegando a Japón) (Cramp, 1985). Su distribución en Europa, no obstante, está fragmentada y se limita en la actualidad a las montañas más importantes del continente (e.g. Pirineos, Alpes, Apeninos) (Hagemeijer \& Blair, 1997, Tucker \& Heath, 2004).

En el caso de España, la especie se cita exclusivamente en el Pirineo Navarro y, vestigialmente, en Huesca (Campion \& Senosiain, 2003). En Navarra la especie ocupa hayedos en buen estado de conservación, tanto puros como mezclados con abeto u otras 
frondosas, desde Belagua hasta el margen occidental de la Zona Especial de Conservación (ZEC) Belate (Fernández et al., 1994). Para el caso de Quinto Real/Kintoa, el pico dorsiblanco ocupa masas de hayedo maduro con extensiones que rondan las 30 ha como mínimo (Garmendia et al., 2006). En ocasiones, existe solapamiento con territorios de picamaderos negro Dryocopus martius L., 1758 (Garmendia et al., 2006). El núcleo de cría más importante del pico dorsiblanco en España se sitúa en Irati, al que le siguen Quinto Real/Kintoa, Bértiz, Alto Roncal y el área de Belate (Cárcamo, 2016).

En conjunto, la población de la especie en España se estima en unas 100 parejas (el $98 \%$ de la cual se localiza en Navarra), motivo por el que es el pícido más amenazado del Estado (Campion \& Senosiain, 2003). Por ello, está catalogado como "En Peligro" a escala nacional y "en Peligro de Extinción" en Navarra (Decreto Foral 563/1995). En Europa está incluido en el Anexo II de la Directiva Hábitats 92/43/CEE, para cuya conservación se establece la necesidad de mantener su hábitat natural.

En este contexto, es importante tanto actualizar el estado de conocimiento de la distribución de la especie como de los requerimientos que necesita para criar, con el fin de llevar a cabo, en última instancia, una gestión que como mínimo garantice la estabilidad de la población actual y, en lo posible, promueva su incremento y expansión hacia otras zonas. La caracterización del sustrato que el pico dorsiblanco emplea para criar forma parte esencial de este conocimiento. Gracias al seguimiento de la nidificación de la especie en Navarra durante los últimos años, se ha podido recolectar gran cantidad de datos sobre el sustrato de nidificación y parámetros reproductivos (tales como fecha y tamaño de puesta, productividad y razón de sexos de la nidada). El objetivo de este artículo es analizar toda esa información con el fin de (1) actualizar y contribuir al conocimiento de la nidotópica de la especie en Navarra, (2) describir su reproducción y (3) proponer, en relación a los resultados que se exponen, medidas de gestión forestal útiles para la conservación de esta especie.

\section{Material y métodos}

\section{Área de muestreo y recolección de información}

El área de muestreo abarca prácticamente todo el área de distribución de la especie en Navarra; concretamente Irati, Quinto Real/Kintoa y Belate, que se incluyen en las respectivas ZEC de Roncesvalles-Selva de Irati (ES0000126), Alduides (ES2200019) y Belate (ES2200018). En todos los casos, se trata de hayedos cuya altitud varía en un rango de $975 \mathrm{~m} \mathrm{s.} \mathrm{n.} \mathrm{m.} \mathrm{a} 1139 \mathrm{~m} \mathrm{~s}$. n. m., en los que dominan las pendientes del $20 \%$ al 40\%, la precipitación es de 1500 a $2000 \mathrm{~mm}$ anuales y el ombroclima es de tipo húmedo o hiperhúmedo, con una fuerte influencia oceánica. 
El estudio se realizó durante el periodo 2003-2018. El muestreo consistió en la localización de nidos durante el periodo de reproducción (marzo-junio), tras lo cual se midieron las siguientes variables, para cada nido activo (ocupado): tipo de sustrato (cuatro categorías: árbol verde y aparentemente sano; puntiseco; mitad verde y mitad seco; seco); orientación y altura de la boca del nido, diámetro y altura del sustrato así como la orientación de la ladera y altitud sobre el nivel del mar. En cuanto a reproducción, cuando se pudo se midió la productividad (i.e. número de pollos que son cebados en el nido, que salen del nido -si se ve el grupo familiar fuera- o en el momento de salir de éste) y se determinó el sexo de los pollos. Para esto se tuvo en cuenta la coloración de la zona superior de la cabeza (rojo en machos, negro en hembras) (Cramp, 1985). Adicionalmente, se midió el tamaño de la cavidad de nidificación en dos nidos que fueron derribados por el viento: altura de la boca del nido, profundidad y anchura de la base del nido.

Durante el periodo de estudio, a cada nido se le asignó un código triple (ID nido, ID tronco, ID territorio). La identificación de los territorios fue establecida al confirmar la excavación del nido por parte de la pareja reproductora y, posteriormente, durante el periodo de seguimiento de la reproducción. Hay que destacar que durante este estudio los individuos no fueron marcados mediante anillamiento u otra técnica para permitir su identificación individual. En caso de territorios previamente desconocidos, cada nuevo nido se asignó a un nuevo territorio

\section{Análisis estadísticos}

En cuanto a estadísticos descriptivos, la media se acompaña de la desviación estándar $(D E)$ y del coeficiente de variación (CV).

Para determinar la existencia de diferencias significativas en proporciones (sustrato de nidificación, razón de sexos en la pollada) se empleó un test de chi-cuadrado $\left(\chi^{2}\right)$.

Para determinar si la orientación de los nidos fue uniforme o no (esto último implicaría la existencia de una o más direcciones preferentes) se empleó un test de Rayleigh. Por otro lado, para determinar si la orientación de la ladera varió significativamente de la de los nidos se llevó a cabo un Análisis de la Varianza para datos circulares. Para ello se empleó el paquete "circular" (Agostinelli \& Lund, 2017) para R (R Core Team, 2014).

Por otro lado, para determinar si las características del sustrato y localización de los nidos varió entre las zonas de estudio se realizó un Análisis Factorial Múltiple (MFA) para variables mixtas (esto es, mezcla de cuantitativas y cualitativas), con las siguientes variables: tipo de sustrato; altura de la boca del nido, diámetro y altura del sustrato y altitud. No se incluyeron factores adicionales ni aleatorios debido al reducido tamaño muestral. Además, y por esa misma razón, sólo se incluyeron Belate y Quinto Real en este análisis. Para realizar este análisis se utilizó el paquete "factominer" (Lê et al., 2008) para $R$. 


\begin{tabular}{|lcccc|}
\hline Año & Aezkoa & Belate & Quinto Real & Salazar \\
\hline 2008 & - & - & 1 & - \\
\hline 2009 & - & - & 1 & - \\
\hline 2010 & - & - & 1 & - \\
\hline 2011 & - & - & 5 & - \\
\hline 2012 & - & - & 4 & - \\
\hline 2013 & - & - & 1 & - \\
\hline 2014 & 1 & - & 5 & - \\
\hline 2015 & - & 7 & 3 & - \\
\hline 2016 & - & 3 & - & - \\
\hline 2017 & - & - & - & 1 \\
\hline 2018 & 2 & 4 & - & 2 \\
\hline Total & 4 & 14 & 21 & 2 \\
\hline
\end{tabular}

Tabla 1.- Número de nidos controlados según zona y año para determinar la productividad de pico dorsiblanco en Navarra, durante el periodo 2008-2018.

Table. 1.- Number of nests monitored depending on zones and years to determine the productivity of the white-backed Woodpecker in Navarra, during the period 2008-2018.

La relación de nidos monitorizados según zona y año se indica en la Tabla 1. Para determinar si la productividad varió entre zonas se llevó a cabo un modelo mixto lineal generalizado (GLMM) con el número de pollos/nido como variable respuesta, la zona como factor y el territorio como factor aleatorio. En este modelo se empleó un enlace log-lineal, con una distribución de errores de Poisson, dada la naturaleza de la variable respuesta (conteo de pollos). El año no fue incluido como otro factor aleatorio debido al reducido tamaño muestral (hubo muchos años para los que $n=1$; Tabla 1). Igualmente, se desistió de tratar de determinar si la productividad varió significativamente entre años. El bajo tamaño muestral en Aezkoa $(n=4)$ y Salazar $(n=2)$ obligaron a incluir en el análisis sólo Belate y Quinto Real.

\section{Resultados}

\section{Nidotópica}

En conjunto, se localizaron un total de 68 nidos. No hubo repetición de nido en ninguno de los años que duró el muestreo. Todos estos nidos se localizaron en 63 árboles, asociados a 33 territorios. En 5 ocasiones (6\%), el nido se ubicó en el mismo pie (árbol) que en años anteriores. El número de nidos observados por territorio varió de 1 (22 territorios) a 8 (1 territorio). 
En 12 casos, se pudo determinar la distancia entre nidos de un año al siguiente, que fue, en promedio, de $115 \pm 128 \mathrm{~m}$ (rango: 0-370 m).

En cuanto al sustrato de nidificación (documentado en 56 pies), todos los nidos se localizaron en haya, en pies puntisecos o totalmente verdes (46\%), hayas secas (40\%) $y$, finalmente en hayas mitad secas-mitad sanas (14\%). Estas proporciones, no obstante, no variaron significativamente entre ellas $\left(\chi^{2}=7,4\right.$; grados de libertad $g l=3 ; P=0,06)$, posiblemente debido a un tamaño muestral relativamente reducido.

En promedio, la altura de los pies con nido fue 19,1 $\pm 8,1 \mathrm{~m}$ (rango: 7,0-32,5 m, $n=29, C V=42,5 \%$ ). La altura de los nidos fue, por otro lado, de 11,1 $\pm 4,2 \mathrm{~m}$ (rango: 3,4-21,0 m, $n=49, C V=37,8 \%$; para los pies con dos nidos se consideró la media). En términos porcentuales, la altura desde el suelo respecto a la altura del árbol fue de un 60,1 $\pm 17,7 \%$ (rango: $18,2-96,7 \%, n=29, C V=29,5 \%$ ). En cuanto al diámetro del tronco, éste varió entre 26,0 y $67,5 \mathrm{~cm}$ (promedio $=42,9 \pm 10,5 \mathrm{~cm}, n=43$, $C V=24,4 \%)$.

En 2015 y 2016, dos de los pies fueron derribados debido al viento, lo que dio la oportunidad de medir las dimensiones del nido: altura de la boca del nido, 6,0 cm en ambos casos; profundidad, 34,0-30,0 cm; anchura de la base del nido, 10,5-11,0 cm. En la base del nido se acumulaba serrín.

En promedio, la altitud de los nidos se situó a 943,8 \pm 99,0 m s.n.m. (rango: 7701180 m). La orientación de las laderas fue variable sin una orientación claramente dominante (test de Rayleigh $=0,186 ; P=0,26$ ). Por el contrario, la orientación de los nidos fue heterogénea (test de Rayleigh $=0,273 ; P=0,05$ ), siendo en este caso el promedio de $180 \pm 92^{\circ}$. La orientación media entre ambos grupos varió significativamente $(F=34,6 ; P<0,001)$.

En el MFA se obtuvieron un total de tres ejes con un autovalor $>1$ (eje 1: 2,84 y 35,5\% de la varianza; eje 2: 1,84 y 22,8\% de la varianza; eje 3: 1,04 y 13,0\% de la varianza), que explicaron el $71,4 \%$ de la varianza. El sustrato donde se ubicaron los nidos en Quinto Real y en Belate varió significativamente entre ambas zonas (Fig. 1). Concretamente, observamos ausencia de solapamiento casi total entre ambas zonas, lo que se explicar por un número mayor de nidos ubicados en pies puntisecos en Quinto Real y en pies mitad verde-mitad seco en el caso de Belate (Fig. 2). Por otro lado, en el caso de Quinto Real los nidos que se pudieron incluir en el estudio se situaron en árboles más gruesos, altos y a mayor altitud, y el nido se situó a mayor altura en el árbol que en los nidos de Belate (Fig. 2).

\section{Parámetros reproductivos}

La productividad se midió en un total de 41 nidos. En la mayor parte de ellos (57,5\%) voló un único pollo (Fig. 3; promedio: 1,4 0 0,7 pollos/nido; rango =0-3). La produc- 


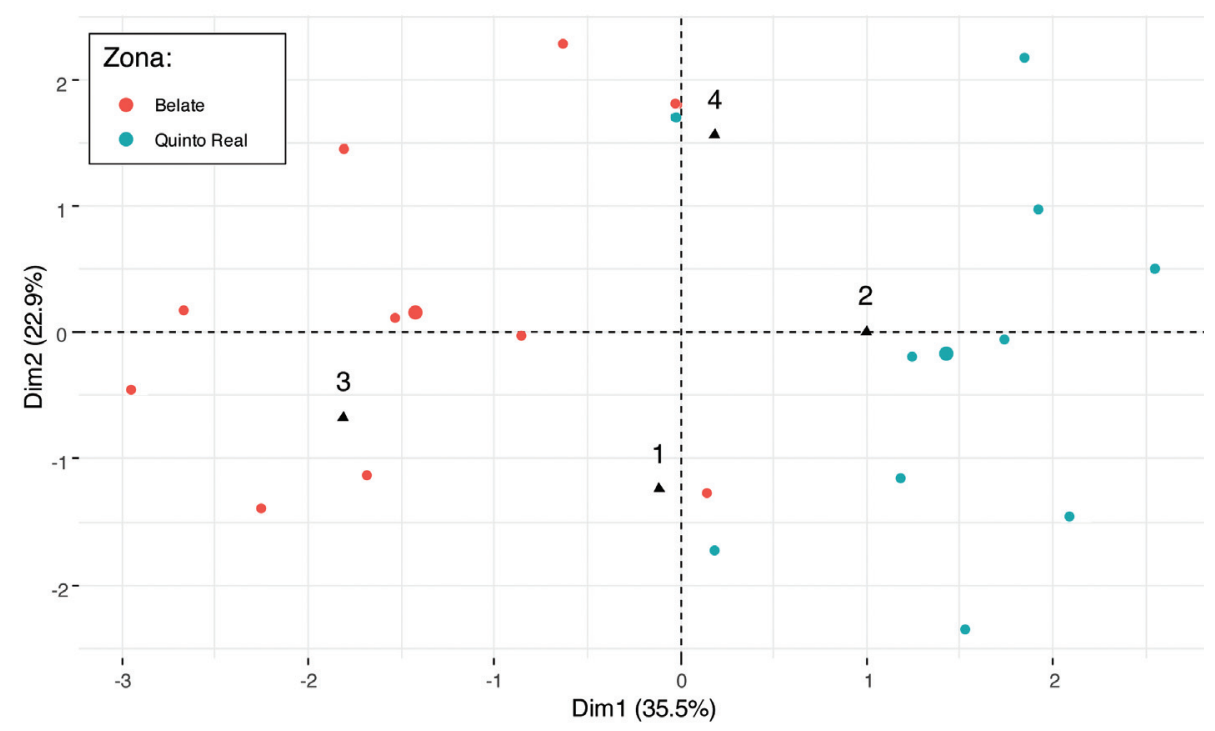

Fig. 1.- Diagrama de las dos primeras dimensiones resultantes de un Análisis Factorial Múltiple para explorar la relación entre variables entre los territorios que se encontraron en Belate y en Quinto Real. Triángulos: tipo de sustrato ( 1 = árbol verde y aparentemente sano; 2 = puntiseco; $3=$ mitad verde y mitad seco; 4 = seco ó snag). Para cada zona, los círculos más grandes indican el promedio.

Fig. 1.- Diagram of the first two dimensions resulting from a Multiple Factorial Analysis to explore the relationship between variables between the territories founded in Belate and those in Quinto Real. Triangles: type of substrate ( $1=$ green and apparently healthy tree, $2=$ trees with dry branches, $3=$ half green and half dry, $4=$ dry or snag). For each zone, the larger circles indicate the average.

tividad no varió entre las dos zonas (Belate y Quinto Real) en las que se obtuvo un tamaño muestral suficiente (efecto zona: $z=-0,070, F=0,005, P=0,945$ ).

Entre 2013 y 2016, se observaron 4 casos de mortalidad en pollos: 3 debido a depredación, 1 debido a mortalidad natural juvenil en un ejemplar a punto de saltar del nido que coincidió con una fuerte bajada de temperatura.

En promedio, la fecha de salto de los pollos del nido fue el 28.05 ( \pm 8 días; rango: 03.05-13.06). Habida cuenta de que el periodo de incubación es de 25-28 días (obs. pers.), la fecha media de puesta se situaría en torno al 01.05-03.05. Se constató que entre el salto del primer pollo y el resto pudo pasar al menos un día, en cuyo caso uno de los progenitores acompañaba al volantón mientras que el otro atendía al (o los) que estaba aún en el nido.

La razón de sexos en los pollos fue de 21 machos/16 hembras (esto es, 1,3 machos/hembra). Este valor no varió significativamente de un ratio $1: 1\left(\chi^{2}=0,68\right.$; $g l=1 ; P=0,40)$. 


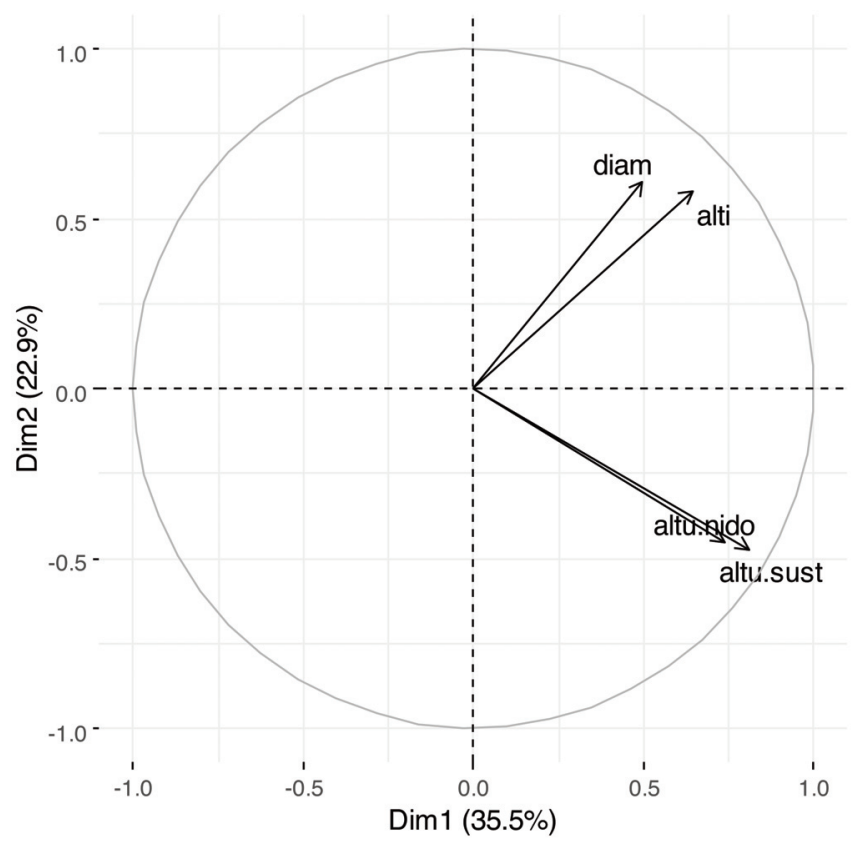

Fig. 2.- Diagrama de las dos primeras dimensiones resultantes de un Análisis Factorial Múltiple para explorar la relación entre variables entre los territorios que se encontraron en Belate y en Quinto Real. En este caso se representan las variables cuantitativas. Abreviaciones: diam = diámetro del pie; alti = altitud; altu.nido = altura de la boca del nido; altu.sust = altura del pie (árbol).

Fig. 2.- Diagram of the first two dimensions resulting from a Multiple Factorial Analysis to explore the relationship between variables in the founded territories in Belate and in Quinto Real. In this case, quantitative variables are represented. Abbreviations: diam = diameter of the tree; alti = altitude; height $=$ height of the nest in the tree; altu.sust $=$ height of the tree.

Fig. 3.- Distribución de frecuencia de la productividad de pico dorsiblanco en Navarra.

Fig. 3.- Distribution of the frequency of the White-backed Woodpecker productivity in Navarra. (Frequency/ Productivity (chicks/nest).

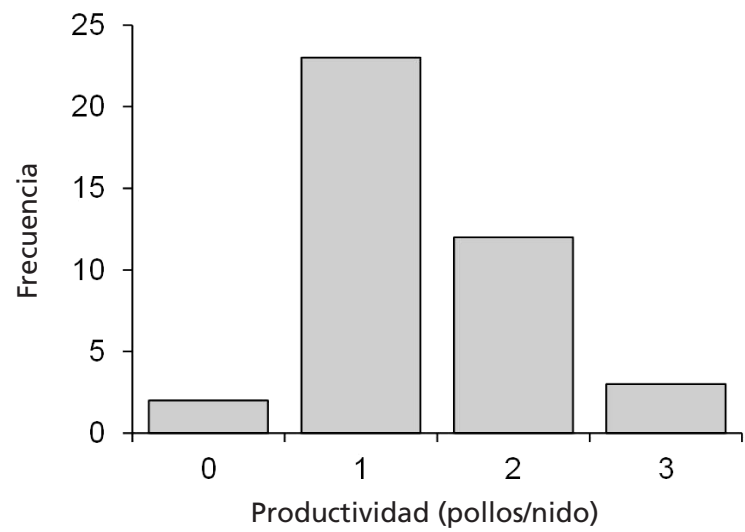




\section{Discusión}

\section{Nidotópica}

Aunque la disponibilidad de pies secos total o parcialmente no se estimó, sí es cierto que la cantidad de hayas secas o, globalmente, pies secos total o parcialmente, no alcanza el $54 \%$ de los pies que hay en el bosque, por lo que sí cabe establecer que existe selección positiva hacia árboles secos, total o parcialmente. En este contexto, la existencia de árboles muertos en el bosque es fundamental para la conservación de este pícido.

Suponemos que los árboles muertos o dañados son más fáciles de perforar y por eso son tan utilizados para la construcción del nido. Debemos considerar, además, que las hayas que hemos considerado sanas pueden estar también dañadas en su interior, pues a partir de $50 \mathrm{~cm}$ de diámetro es habitual el desarrollo de la enfermedad denominada "corazón rojo", una pudrición del duramen causada por un hongo y que, con frecuencia, no se manifiesta externamente. En este contexto, se ha descrito que los picamaderos negros seleccionan hayas aparentemente sanas para instalar sus nidos que, a menudo, presentan esta enfermedad (Zahner et al., 2012), lo que sugiere que el caso del pico dorsiblanco podría ser parecido.

En el caso de Navarra todos los nidos se ubicaron en haya, resultados que coinciden con lo descrito para Francia, donde el $\mathbf{9 7 \%}$ de los nidos fue localizado en haya. En este último caso, debe considerarse que en la cara septentrional de Pirineos el hayedo abetal es más extenso que en la cara sur, por lo que la preferencia por el haya es bastante clara (Grangé, 2015). Como en otras zonas (Gorman, 2004, Grangé, 2015), en Navarra el pico dorsiblanco construye un nido nuevo cada temporada, normalmente en un nuevo árbol, si bien a veces puede utilizar el mismo árbol varios años.

El pico dorsiblanco prefiere un sustrato liso y excava el nido en una zona sin ramaje con el fin de facilitar la entrada y salida y evitar la depredación. En el caso de Navarra, los nidos se localizaron a unos $11 \mathrm{~m}$ sobre el suelo, lo que en promedio es el $60 \%$ de la altura total del árbol desde el suelo. Esta altura se sitúa en el rango dado para otras zonas de Europa (e.g., Grangé et al., 2002). Parece ser que las martas Martes martes L., 1758 son uno de los depredadores con más impacto en la especie, sobre todo en nido (Purroy, 2014). En Navarra, se constataron al menos tres casos de depredación en los que la entrada al nido había sido abierta a mordiscos, tal y como proceden las martas, para depredar pollos.

La orientación de los nidos fue heterogénea, siendo el promedio de $180^{\circ}$, esto es, orientación sur. Esto contrasta con los resultados de Francia, donde los nidos se orientan, principalmente, hacia el norte (Grangé, 2009). Esta diferencia, aparentemente, no se debe a la orientación dominante de las laderas que, al menos en Navarra, presentaron una orientación media distinta a la observada en nidos. Según Landler et al. 
(2015), no obstante, para determinar si la orientación de la cavidad de un nido es o no aleatoria es necesario un tamaño muestral superior a 100 nidos, del que todavía estamos muy lejos. Sobre la distancia entre nidos en años subsecuentes, los resultados que se obtuvieron para Navarra no difieren de lo reportado para el Pirineo Francés (Grangé, 2009), donde los nidos de años anteriores se alejan entre ellos entre 0 y $300 \mathrm{~m}$.

Un Análisis Factorial Múltiple reveló diferencias significativas entre las zonas de Belate y Quinto Real en lo relativo a las características del árbol donde se localizó el nido. Esto supone cierta flexibilidad a la hora de adaptarse a las condiciones específicas de cada masa forestal, dentro de los rangos que se han descrito arriba. Esto implicaría que la especie es capaz de explotar diferentes tipos de masas forestales, con un porcentaje variable de hayas secas y pies de distinto tamaño (esto es, hasta cierto punto, con distintos grados intensidad en el aprovechamiento forestal), lo que a su vez establece un escenario real para el desarrollo de una política forestal que sea sostenible con la conservación de la especie.

En cuanto a productividad, la población de Navarra tuvo un promedio de 1,4 pollos/nido, que es una de las productividades más bajas que se han registrado en Europa: Noruega, 2,4 pollos/nido (Hogstad \& Stenberg, 1997); Finlandia, 2,8 pollos/nido (Lehikoinen et al., 2013); Polonia, 3,4 pollos/nido (Wesolowski, 1995). A priori, este resultado hace pensar en que la reproducción de este pícido en Navarra es subóptima. Hay muchos factores que influyen en la reproducción de esta especie pero el estado de la hembra, dependiente de la calidad del hábitat, parece ser muy importante (Hogstad \& Stenberg, 1997). Una productividad baja se debe, a menudo, a un bajo ritmo de cebas, lo que se asocia a hábitats con un escaso número de recursos tróficos (Grangé et al., 2002). En los Pirineos las tasas de ceba son las más bajas de toda Europa (Grangé, 2015), lo que explicaría una productividad tan baja. En este contexto, un factor clave sobre la calidad del hábitat es la cantidad de madera muerta y árboles dañados (Carlson, 1998, Gorman, 2004). Tales recursos se vuelven muy escasos si los tratamientos silvícolas eliminan la madera muerta. Por tanto, desde el punto de vista de la conservación de este pico es esencial mantener toda la madera muerta que genere el bosque, tanto en suelo como en pie. Esta última juega un papel importante en invierno, ya que la madera muerta en pie puede ser explotada cuando la nieve cubre la madera muerta sobre el suelo (Gorman, 2004) y, además, se descompone más lentamente. Las hayas secas situados alrededor de los nidos son explotados muy activamente durante el proceso de ceba de los pollos, presumiblemente porque suministran abundante alimento de calidad. Además, los diámetros más gruesos tienen mayor valor ecológico ya que se descomponen más lentamente y tienen mayor capacidad de albergar larvas de insectos de las que se alimentan gran cantidad de especies, entre ellas los picos. 


\section{Conclusiones y propuestas para la gestión de la especie}

La fecha media de salto registrada para la población de Navarra hace recomendable minimizar las molestias entre los meses de marzo a junio, siendo abril-mayo los meses más críticos (puesta, incubación y cebas). Además, la escasa distancia entre los nidos de años consecutivos indica la existencia de un área núcleo de reproducción reducida, y en consecuencia apunta a la necesidad de respetar el área de nidificación. En este sentido, el Departamento de Medio Ambiente del Gobierno de Navarra ha desarrollado unas directrices de gestión de bosques para el pico dorsiblanco. Debe destacarse, además, la importancia de mantener en pie los árboles con nidos viejos porque éstos pueden ser usados para hacer un nuevo agujero en años subsiguientes. Se propone la aplicación de una gestión del bosque que permita que éste genere su propia madera muerta, sin eliminar los pies muertos o dañados a lo largo de todos los periodos de corta del ciclo de un hayedo, tanto si están en posición vertical como caídos y con independencia del diámetro. En general, se propone dejar pies de bajo valor maderable, cuyo interés para el ecosistema es superior al que puedan reportar económicamente.

\section{Agradecimientos}

Este trabajo ha sido financiado en parte por el Departamento de Medio Ambiente del Gobierno de Navarra. Agradecemos a los Guardas Forestales de las Demarcaciones de Aezkoa- Quinto Real, Roncal-Salazar y Ultzama-Arakil del Gobierno de Navarra los datos sobre los nidos de la especie aportados para la realización de este artículo.

\section{Bibliografía}

Agostinelli, C., Lund, U. 2017. R package "circular": Circular Statistics (version 0.4-93). URL https://r-forge.r-project.org/projects/circular/.

Campion, D., Senosiain, A. 2003. Pico dorsiblanco, Dendrocopos leucotos. In: Atlas de las aves reproductoras de España. R. Martí and J. C. Del Moral (Eds.): 360-361. DGCN-SEO/BirdLife. Madrid.

Cárcamo, S. 2016. Distribution du Pic de Lilford Dendrocopos leucotos lilfordi à l'ouest des Pyrénées espagnoles. Le Casseur d'os 16: 96-104.

Carlson, A. 1998. Territory quality and feather growth in the White-Backed Woodpecker Dendrocopos leucotos. J. Avian Biol. 29: 205-207.

Cockle, K. L., Martin, K., Wesolowski, T. 2011. Woodpeckers, decay, and the future of cavitynesting vertebrate communities worldwide. Frontiers in Ecol. Environ. 9: 377-382. 
Cramp, S. 1985. Handbook of the Birds of Europe, the Middle East and North Africa. Vol. 4. Oxford University Press. Oxford.

Fernández, C., Azkona, P., Llorente, L. 1994. Corología y caracterización del hábitat del pico dorsiblanco (Dendrocopos leucotos) en el pirineo occidental español. Ardeola 42: 135-140.

Garmendia, A., Cárcamo, S., Schwendtner, O. 2006. Forest management considerations for conservation of Black Woodpecker Dryocopus martius and White-backed Woodpecker Dendrocopos leucotos populations in Quinto Real (Spanish Western Pyrenees). Biodivers. Conserv. 15: 1399-1415.

Gorman, G. 2004. Woodpeckers in Europe. A study of the European Picidae. Bruce Colleman Books. Chalfont St Peter.

Grangé, J.-L. 2015. Breeding biology of the Lilfordi Woodpecker Dendrocopos leucotos lilfordi in the Western Pyrenees (SW France). Denisia 36: 99-111.

Grangé, J. L. 2009. Caractéristiques des arbres de nid chez le pic à dos blanc Dendrocopos leucotos lilfordi dans les Pyrénées occidentales françaises. Le Casseur d'Os 9: 92-110.

Grangé, J. L., Auria, J. C., André, G., Navarra A, P. 2002. Biologie de reproduction du pic à dos blanc (Dendrocopos leucotos lilfordi). Nos Oiseaux 45: 194-212.

Hagemeijer, W. J. M., Blair, M. J. 1997. The EBCC Atlas of European Breeding Birds. Their distribution and abundance. T \& A D Poyser. London.

Hogstad, O., Stenberg, I. 1997. Breeding success, nestling diet and parental care in the White-backed Woodpecker Dendrocopos leucotos. J.I Fur Ornithol. 138: 25-38.

Landler, L., Justino, M., Skelton, J., Walters, J. 2015. Global trends in woodpeckers cavity entrance orientacion: latitudinal and continental effects suggests regional climate influence. Acta Ornitol. 49: 257-266.

Lê, S., Josse, J., Husson, F. 2008. FactoMineR: An R Package for Multivariate Analysis. J. Statistical Software 25: 1-18.

Lehikoinen, A., Jaatinen, K., Vähätalo, A. V., Clausen, P., Crowe, O., Deceuninck, B., Hearn, R., Holt, C. A., Hornman, M., Keller, V., Nilsson, L., Langendoen, T., Tománková, I., Wahl, J., Fox, A. D. 2013. Rapid climate driven shifts in wintering distributions of three common waterbird species. Global Change Biol. 19: 2071-2081.

Purroy, F. 2014. Picamaderos negro - Dryocopus martius.in A. Salvador and M. B. Morales, editors. Enciclopedia Virtual de los Vertebrados Españoles. Museo Nacional de Ciencias Naturales. Madrid.

R Core Team. 2014. R: A language and environment for statistical computing. ISBN 3900051-07-0. Vienna, Austria.

Roberge, J.-M., Mikusi ski, G., Svensson, S. 2008. The white-backed woodpecker: umbrella species for forest conservation planning? Biodivers. Conserv. 17: 2479-2494. 
Cárcamo et al.

Sekercioglu, C. H. 2006. Increasing awareness of avian ecological function. Trends in Ecol. Evol. 21: 464-471.

Tucker, G. M., Heath, M. F. 2004. Birds in Europe: population estimates, trends and conservation status. BirdLife International. Cambridge.

Wesolowski, T. 1995. Ecology and behaviour of White-backed Woodpecker Dendrocopos leucotos in a primeval temperate forest (Bialowieza National Park, Poland). Die Voge/warte 38: 61-75.

Zahner, V., Sikora, L., Pasinelli, G. 2012. Heart rot as a key factor for cavity tree selection in the black woodpecker. Forest Ecol. Manag. 271: 98-103.

Fecha de recepción/ Date of reception: 05/02/2018

Fecha de aceptación / Date of acceptance: 11/12/2018

Editor Asociado / Associate editor: David Álvarez 